\title{
Motivate students to recall the concepts in the prerequisites prior to the online lectures.
}

\author{
Eng. Sundaresh Jeyaram ${ }^{\mathrm{a}}$, Eng. Mohammed Suhail ${ }^{\mathrm{b}}$, Mr. S. Loheeswaran ${ }^{\mathrm{c}}$ \\ ajeyarams@esn.ac.lk \\ ${ }^{a}$ Eastern University, Sri Lanka, Nilaveli, Trincomalee, 31010, Sri Lanka \\ ${ }^{\mathrm{b}}$ suhailmpm@esn.ac.lk \\ ${ }^{b}$ Eastern University, Sri Lanka, Nilaveli, Trincomalee, 31010, Sri Lanka \\ cloheeswarans@esn.ac.lk \\ ${ }^{c}$ Eastern University, Sri Lanka, Nilaveli, Trincomalee, 31010, Sri Lanka
}

\begin{abstract}
In online teaching and learning systems it is important to utilise the time and resources effectively, as well as the students' attainments also need to be evaluated transparently. Here two major problems arise for the teacher while delivering online due to COVID 19; Students lose the Low Order Thinking Skills (LOTS) in the prerequisites. In the other hand, assessing formative assessments with fair scale. Because assessment methods are not transparent as students are not aware how they are going to be assessed. This study focused to suggest and examine effective solutions for assisting students to recall the concepts in the prerequisites with transparent analytic scoring rubrics assessment technique and make the online teaching more effective. Pre-lecture resources with a presentation task as an achievement motivation were suggested as a solution for this problem. It was implemented to a class of 30 students who are in the $2^{\text {nd }}$ year, following engineering degree in Sri Lanka. The results were attained from the students' and teachers' feedbacks, as well as from the students' achievements from the scoring rubrics. Hence, it was observed that the students were highly motivated and teacher spent lesser time in delivering online sessions.
\end{abstract}

Keywords: Achievement Motivation; LOTS; Scoring Rubrics

\section{Introduction}

In the general practice, it has been identified that the students forget the concepts studied in the prerequisites, so they take lot of time to grab the new concepts taught in the current lectures. It leads to an inefficient lecturing time by repeating the concepts that they already learnt. Pre-lecture resources with a presentation task as an achievement motivation were suggested as a solution for this problem. It also give a great opportunity to the students to achieve the Low Order Thinking Skills (LOTS) before coming to the lectures.

It was implemented to the $2^{\text {nd }}$ year students who are following the module Electronic Circuits in the School of Engineering and Technology in Sri Lanka. The module has two prerequisites those are Electrical Properties of Materials and Digital Electronics II, and followed by 30 students. 
In case of assessing students' performance in their presentation, old assessment methods were found to be not transparent, and students had difficulty in identifying what sort of task or criteria they had been set. Therefore an alternative approach was used to assess the presentations.

\section{Research Elaboration}

To help students for recalling the concepts of prerequisites, the suggested method is providing pre-lecture sources prior to the lectures and let each student make individual presentations on a specific topic in prerequisites. These presentations were conducted as a part of formative assessment for the current module.

Pre-lecture resources are a way of scaffolding students' learning of the new material in a module by providing in advance of each lecture some information to ease the cognitive load associated with new material presented in a lecture [1]. Pre-lecture resources aim to reduce intrinsic load by introducing some terminology or concepts prior to a lecture, so that students can become familiar with these and allow for working memory in the lecture to concentrate on integrating these terms/concepts into long term memory.

For assessing presentations following approach was applied. The assessing scheme and defined criteria was disclosed and discussed with the students prior to the presentation. Then Students' feedback were considered for assessment after the presentation.

\section{Results}

\subsection{Issuing Pre-Lecture sources prior to the lectures}

The results were evaluated from the students' performance in their presentation, by observing the delivery time taken for the next lecture, and by getting the students' feedback.

Because of the presentation activity, students looked so curious and more energetic in the lecturing day, not only that but also the time taken for the lecturing was reduced by 45 minutes which is quite significant amount. The effective use of the lecturing time helped the students as well as the lecturer to keep the class more active rather than getting tired or bored.

\subsection{Assessing students in a transparent approach}

The results were evaluated from the students' performance and the quality of their presentation, students' achievements of ILO through the presentation, and by getting the students' feedback.

23 students did the presentation out of 30 students. The results of the students' overall performance has been shown in Fig. 1: as 1 - poor; 5 - excellent. 


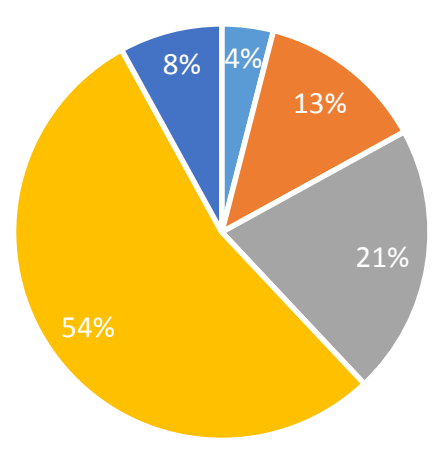

$=1-2=3-4=5$

Fig. 1. Students' overall performance

The one of the module's ILOs "Identify different amplifier configurations and demonstrate the operational aspects" was assessed by having a viva right after the students' presentation activity. The students' achievements of ILO has been shown in the Fig. 2.

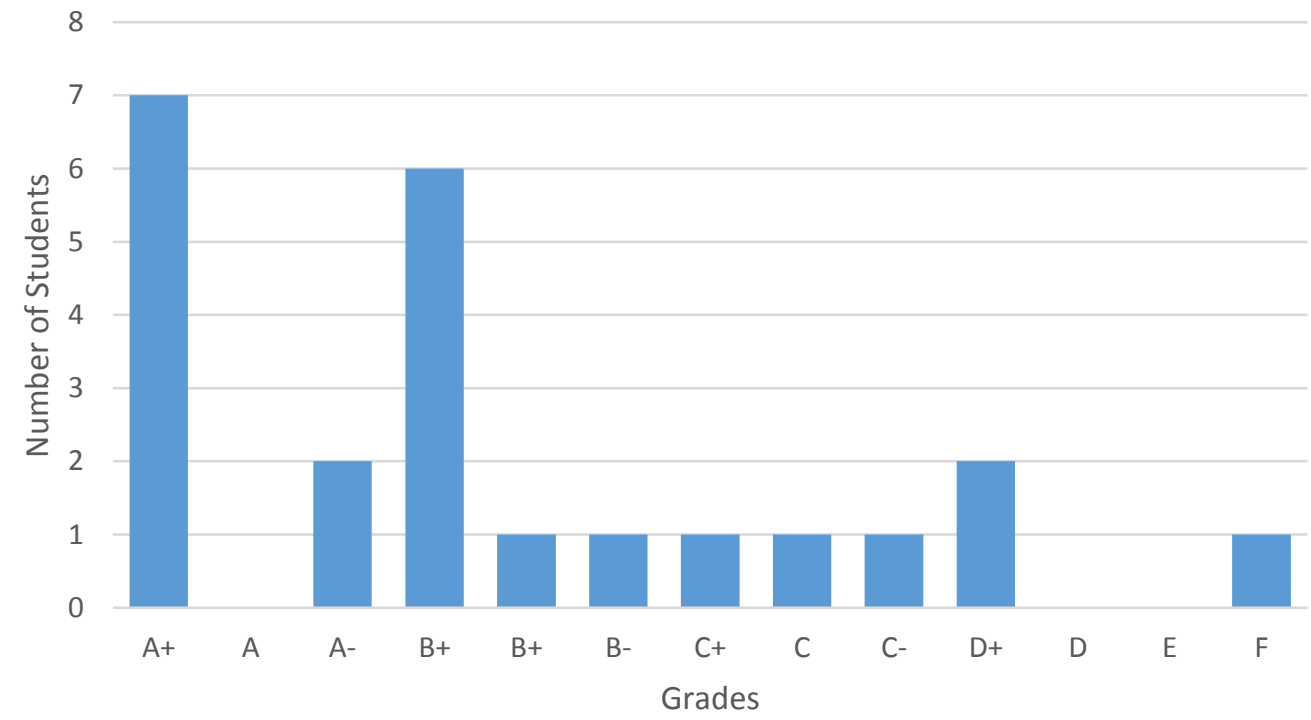

Fig. 2. Students' results on the ILO

Students' feedback were taken for both cases (Issuing Pre-Lecture sources prior to the lectures. And assessing students in a transparent approach). It was observed that he students' responses to the feedback increased from $73 \%$ to $100 \%$, from the previous cases. 


\section{Conclusion}

Students were encouraged to recall the gathered knowledge in the prerequisites using effective pre-lecturing resources as well as a presentation activity which acted as an achievement motivation.

The outcomes were evaluated through students' performance in their presentation activity, time taken to deliver the lecture, and finally the students' feedback. From the results of the above evaluations, it can be concluded that the end result was quite remarkable.

The online based presentation activity as formative assessment with a transparent scoring rubric method of evaluation was implemented and evaluated through students' performance and the quality of their presentation, students' achievements of ILO through the presentation, and finally the students' feedback. From the results of the above evaluations, we could observe that the end result was quite remarkable.

\section{Acknowledgments}

We would like to express our gratitude to the Staff Development Center of Eastern University, Sri Lanka for their support and guidance for this teaching, learning and assessment approach.

\section{References}

[1] Michael K Seery, Using Pre-Lecture Resources in your Teaching: A Short Guide, https://www.academia.edu/2839558/Using Pre-Lecture_Resources_in_your_Teaching_A_Short_Guide, accessed: August, 2021.

[2] Graham Gibbs, Trevor Habeshaw, Preparing to Teach, Technical and Educational Services Limited, 1989, Ch. 4. 